\title{
A genetic test for androgenetic alopecia: polymorphisms in the androgen receptor gene provide a genetic screening test for androgenetic alopecia and earlier medical intervention
}

\author{
Sharon A. Keene, MD Tucson, Arizona
}

\section{Introduction}

Although the comprehensive details of genetic inheritance for androgenetic alopecia (AGA) are not yet characterized, prevailing evidence supports a polygenic model based primarily on the fact that autosomal and single gene inheritance theories are not supported by observed patterns. Evidence against an autosomal pattern is the observed phenotypic prevalence of AGA in families, which does not fit the expected bimodal distribution. A single gene hypotheses would yield an expected prevalence of around 1:1000, far less than the high percentage of affected males seen in population studies. ${ }^{1}$ Efforts to identify the involved genes have focused on those known to be related to mechanisms of hair growth. However, tests failed to reveal any association between the suspect genes and AGA, with one notable exception. There have been four published studies involving over 2,900 patients that document the association between AGA and the androgen receptor (AR) gene on the $\mathrm{X}$ chromosome. A particular polymorphism or variant allele at the AR site has been shown to be associated with a higher risk of developing AGA ( $>60 \%$ ) and, conversely, a protective, less frequent allele has been associated with a very low likelihood of developing AGA ( $>85 \%$ probability of not developing AGA). In order to assess the usefulness of this finding, we must first distinguish between what is meant by a "screening test" versus "diagnostic test." The advantages of a screening test to identify a high-risk population for AGA, prior to visible signs of hair loss, for the purpose of early medical intervention will be discussed.

\section{Background}

In distinguishing the difference between a screening test and a diagnostic test, it is important to realize that a screening test is designed to indicate a risk or probability of developing a disease or condition prior to the onset of symptoms. ${ }^{2}$ In contrast, a diagnostic test is designed to confirm a suspected diagnosis based on the presence of symptoms. Screening tests may lead to diagnostic or confirmatory tests to establish a suspected diagnosis. One objective of screening tests, by identifying the condition prior to the onset of symptoms, is to make the diagnosis in an earlier or preclinical stage, so that treatment can be initiated at a time when intervention has a greater likelihood of success. The criteria for a good screening test is that the condition being screened should have a high prevalence in the population being screened, and should result in treatment of the condition before the onset of symptoms. ${ }^{2}$ Finally, a good screening test has a level of sensitivity and specificity that correlates with the severity of the condition being screened such that false positives and false negatives do not place patients in jeopardy with regard to over or under diagnosis. In the case of androgenetic alopecia, studies have shown a high prevalence of AGA with increasing phenotypic expression based on advancing age, so that the majority of men will be affected by the age of $80 .^{\prime}$ Current diagnosis relies largely on the development of a hair loss pattern and visible areas of thinning or baldness. In fact, it has been shown that visible thinning does not occur until there has been approximately $50 \%$ hair loss in a given area. Therefore, a paradigm that depends on the development of a hair loss pattern for diagnosis ensures that patients will have substantial hair loss prior to initiation of therapy. This fact becomes more important when taking into account that the two FDA-approved medication therapies to combat hair loss-minoxidil and finasteride-are most effective at stabilizing hair loss rather than hair regrowth. Neither therapy can re-populate lost hair follicles, and regrowth is limited to strengthening existing vellus hairs. Therefore, a screening test for androgenetic alopecia that identifies patients at higher risk for AGA can offer the opportunity for early medical intervention prior to visible signs of hair loss, when stabilization is most cosmetically beneficial. This satisfies the criteria for a clinical situation where a screening test is useful.

While it is easy to describe the advantage of a screening test for AGA, the challenge has been to identify a gene or marker able to fit these criteria. Before reviewing the studies that provide the basis for the new genetic screen for AGA, it is helpful to review certain predictive characteristics of other well-known screening markers for comparison. Two of these are prostate-specific antigen (PSA) and carcinoembryoic antigen (CEA). PSA is a proteoglycan and has been used to screen for prostrate cancer, despite a reported tissue biopsy rate of nearly $65 \%$ false positives. Furthermore, at least $20 \%$ of men with biopsy proven cancer had a PSA in the reference range of normal. ${ }^{3}$ Despite limitations it has been widely used and credited for an overall decrease in mortality due to earlier diagnosis and therapy. ${ }^{4}$ Two studies are under way to assess this assumption.

Carcinoembryonic antigen was first described in 1965 by Gold and Freeman who identified the antigen in colon adenocarcinonoma, but not in healthy adult colon tissue. Eventually it was found in some normal tissues, too. At a cutoff level of $2.5 \mathrm{microgm} / \mathrm{L}$, sensitivity ranges from $30-80 \%$ depending on the stage of disease. While the positive predictive value of the test is low for primary diagnosis, the test is 
Genetic test for AGA

$\checkmark$ from page 91

considered a good screening tool for prognosis and staging as well as cancer recurrence. ${ }^{4}$ What is apparent from these and other screening tests is that none is a perfect marker, since none are $100 \%$ sensitive or specific nor have a $100 \%$ positive or negative predictive value. It is also worth noting that neither PSA or CEA are causative agents for the cancer they are associated with. A screening marker does not need to be either causative or diagnostic-only associated or predictive of risk.

Androgenetic alopecia is presumed to be polygenic therefore no single gene can be identified as the cause; however, in the past few years, there have been at least four published studies to confirm the association of the androgen receptor gene with AGA. Specific risk assessment has been further classified by identification of polymorphisms of the AR gene. The first study linking the AR gene to AGA compared men with Kennedy disease, an X-linked neurodegenerative disease, caused by a repeat, nucleotide sequence within the AR gene. This appears to create partial androgen insensitivity due to loss of receptor function. Since it is known that AGA is affected by androgens at the level of the hair follicle, one would expect that receptor insensitivity would result in a decrease incidence of AGA. In the reported study, 115 respondents with Kennedy disease were compared to 654 Australian Caucasians and were asked to rank their hair loss based on a weighted Norwood Hamilton scale. Men with Kennedy disease were significantly less likely to experience AGA. Furthermore, age was found to be a highly significant variable in the control group among the men with AGA but not among men with Kennedy disease. ${ }^{6}$

The next three studies specifically looked at the allele polymorphism, which has been used as the basis to create a screening test for AGA. In the first study, several families underwent genotyping. Among them, 198 had early onset AGA; there were 188 controls and 157 who were unaffected. A strong association was found with rs6152 in exon 1 of the AR gene corresponding to the stu 1 restriction fragment length polymorphism $(\mathrm{p}=6.66 \times 10-10) .{ }^{7}$ The Stu 1 restriction site was again studied by Levy-Nissenbaum et al. The Stu 1 restriction site in the AR gene was present in 39 of 41 bald men, and only 26 of 38 non-bald males $(p<0.0026)$ In assessing this allele as a marker for AGA, the Stul restriction or "cut" allele verses the "uncut" allele confirms a $60 \%$ increased risk for AGA. ${ }^{7}$ In a separate study, genomic analysis was performed looking at the same "uncut" A allele for an association with either AGA and/or prostate cancer. This study looked at 815 prostate cancer cases and 719 case controls. The proportion A allele carriers (uncut) was the same for each group. There was no associated risk with the A allele and prostate cancer. However, a strong inverse association was found with A allele carriers and AGA that appeared to be protective against developing AGA. Overall, $81 \%$ of men with frontal baldness, $89 \%$ of men with vertex baldness, and $93 \%$ of men with combined front and vertex baldness did not have the A allele. ${ }^{8}$ This study is in concordance with the previous study, which showed the uncut allele (A) to have an $85 \%$ positive predictive value for not developing AGA.

\section{Discussion}

In analyzing the data from the three studies investigating AR gene polymorphisms and AGA, a statistically significant association was found. The cut allele (G) has a $91 \%$ sensitivity and $31 \%$ specificity to provide a positive predictive value of $60 \%$ for developing AGA. The uncut allele (A) offers an $85 \%$ predictive value of not developing AGA.

The value of this test is that it offers the first credible, reproducible screen for assessing an increased risk for the development of AGA prior to the onset of symptoms. It also provides a means for patients with the A allele, and a strong family history of AGA, to learn their risk of developing AGA is, nevertheless, small. Because of the limitations in specificity at this time, a confirmation test should be used to determine the need for medical therapy. Confirmation tests include the "pull test" or one of the newer trichometric methods of assessing Anagen:Telogen ratio. A normal A:T ratio is 12:1; as AGA progresses, this drops to 5:1.' Documentation of a falling A:T ratio indicates the onset of AGA. Although the sensitivity of the genetic screen is high, the specificity reduces the predictive value, so how is this test better than watchful waiting? First, studies have shown that men are reluctant to visit doctors unless they think that something is "wrong" (2007 Harris Interactive Online Survey for AAFP), and without visible thinning or some other warning, many won't seek medical therapy or advice. Furthermore, many patients shed very gradually; they do not see hair in the sink, on the pillow or on their clothes and thus may not notice hair loss is occurring, once again, until a pattern has developed. Allowing these patients an opportunity to become aware of their risk can increase vigilance, allow them to learn about their options, and cause them to seek more frequent medical assessment until medical intervention is warranted. Conversely, those patients who will suffer rapid shedding, may not do so in a gradual, stepwise pattern of shortened growth cycles, but rather a sudden change from anagen to telogen and then hair loss without gradual miniaturization. This group of patients will need intervention very quickly to intercede in the hair loss process, and in the absence of planning in advance, it is likely that delays in getting in to see their doctor and instituting therapy will result in unnecessary hair loss and perhaps alternate more expedient but perhaps less useful therapies in the meantime.

The continued success of many ineffective, unapproved treatments for hair loss suggests that many patients have not been educated by medical doctors regarding effective treatment options. A relatively inexpensive screening test, which is noninvasive, easy to perform, and highly reproducible, can potentially attract patients to their doctor to seek expert advice. Furthermore, while it is true that some patients who initiate medical therapy may never develop significant hair loss or require future hair restoration surgery, there will be those who do not seek or stay on medical therapy despite their known risks. For example, consider the fact that cholesterol screening and lipid lowering medications while lifesaving for many have not eliminated the need for coronary artery bypass surgery. Likewise, hair restoration surgery will continue, for now, to be the most effective therapy for replacing areas of bald scalp. 


\section{Conclusion}

Polymorphism of alleles in the Stu 1 restriction site in the androgen receptor gene has provided a reasonably predictive screening test for high or low risk for development of AGA. The high-risk variant, coupled with confirmatory tests, provides an opportunity for early medical intervention in AGA prior to significant hair loss at a time when stabilization is most cosmetically effective. The low-risk variant can relieve some anxiety among those who have the protective allele. Additional genetic findings in the future may offer further opportunities to improve the sensitivity, specificity, or even diagnostic capabilities of this test.

\section{References}

1. Sinclair, R., et al. Male androgenetic alopecia. Chapter 18, Endotext.com, July 2007.

2. Herman, C.R., et al. Screening for preclinical disease: test and disease characteristics. John Hopkins Medical Center, American Journal of Roentgenology 2002, 179:825-831.

3. Beduschi, R., Beduschi, M., Oesterling, J. Percent free prostate-specific antigen test: improving both sensitivity and specificity. Infect Urol 1998; 11:133-138.

4. Linn, M., Fall, R.A., Maradiegue, A. Prostate specific antigen screening: friend or foe? Urol Nurs 2007; 27 (6), 481-489.

5. Duffey, M. CEA as a marker for colorectal cancer: is it clinically useful? Chemical Chem 2001; 47:4, 624-630.
6. Sinclair, R., et al. Men with Kennedy disease have a reduced risk of androgenetic alopecia. Brit J of Derm 2007 157, 290-294.

7. Hillmer, A. et. al. Genetic variation in the human androgen gene is the major determinant of common early-onset androgenetic alopecia. American Journal of Human Genetics 2005 (77): 140-148.

8. Levy-Nissenbaum, E., et. al. Confirmation of the association between male pattern Baldness and the androgen receptor gene. European Journal of Dermatology 2005, Vol. 15, No. 5, 339-40.

9. Hayes, V., et al. The E211 G > A androgen receptor polymorphism is associated with a decreased risk of metastatic prostate cancer and androgenetic alopecia. Cancer Epidemiology, Biomarkers and Prevention, April 2005, Vol. 14, 993-996.

Editors' note: Sharon Keene is Chief Medical Officer for Hairdx.com, the company that filed the patent for this AGA genetic test. It is not a blood test; it is a mucosal swab from the inside of the cheek. Patients can order this test online (the price is US \$149), but it can also be offered by physicians.

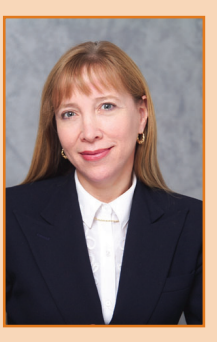
Information about this company, its members and advisors, and how to purchase the test can be found at www.hairdx.com.

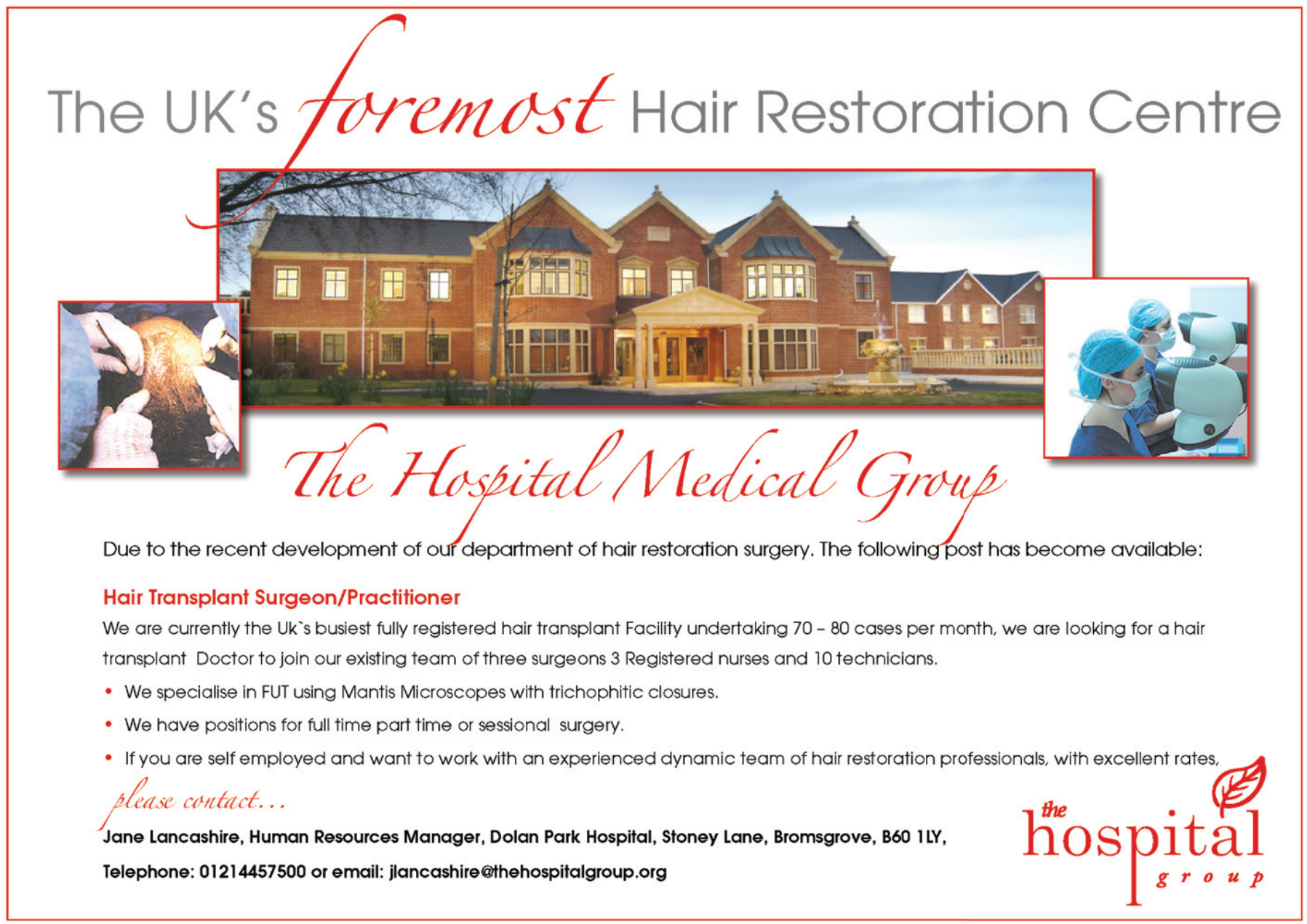

